
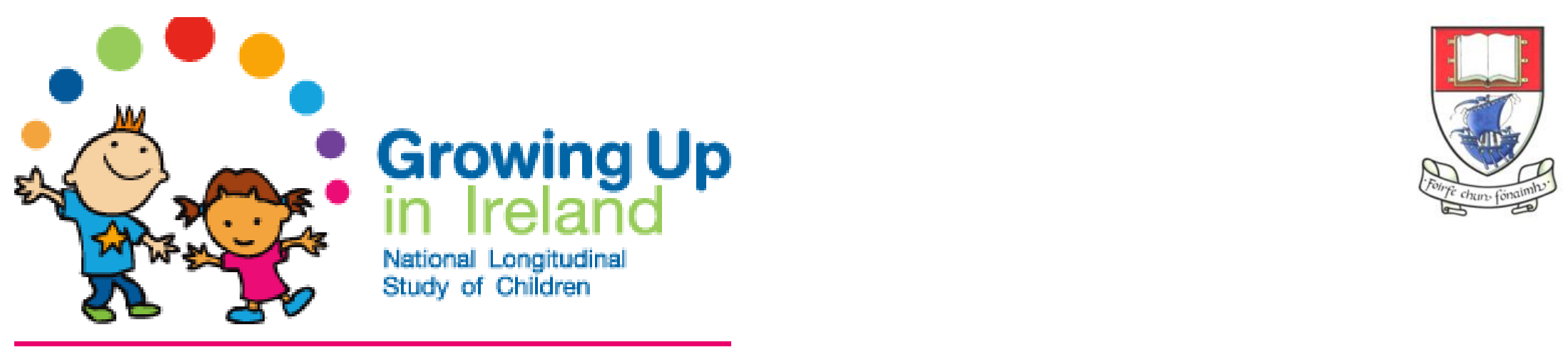

Correlates and Implications of High Screen Time among High and Low Active Irish Nine-

\title{
Year Olds
}

Aoife Lane, Niamh Murphy, Michael Harrison

Centre for Health Behaviour Research, Waterford Institute of Technology, Ireland 
Does screen time(ST) predict overweight/obesity(OW/obesity) independently of physical activity(PA) in 9 year old children?
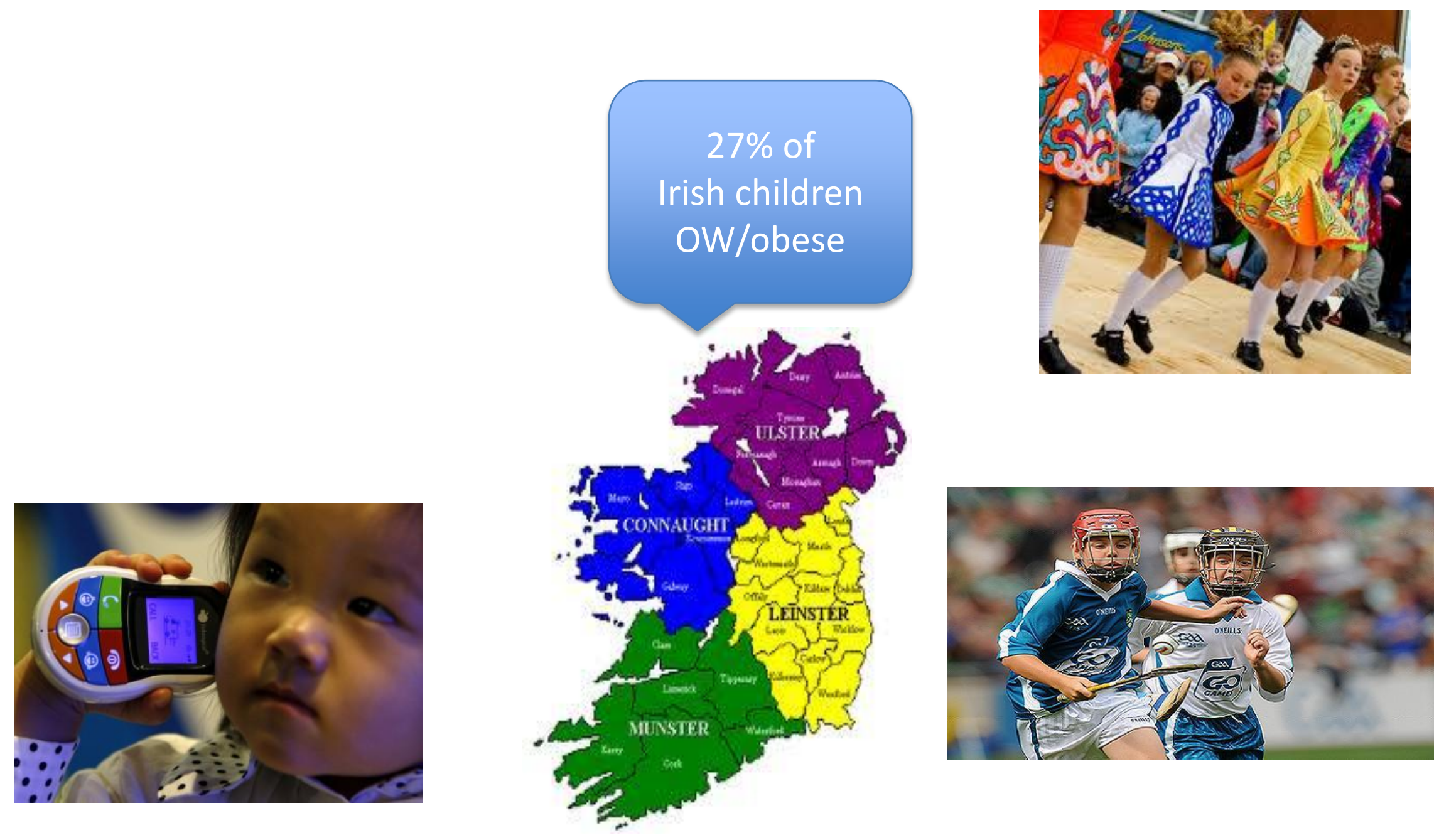


\section{Research Questions}

- RQ1 What is the combined influence of ST and PA on risk of OW/obesity in a large nationally representative cohort of 9 year old Irish school children?

- RQ2 Even in sufficiently active children, is high ST a risk factor for OW/obesity?

- RQ3 What are the correlates of high ST?
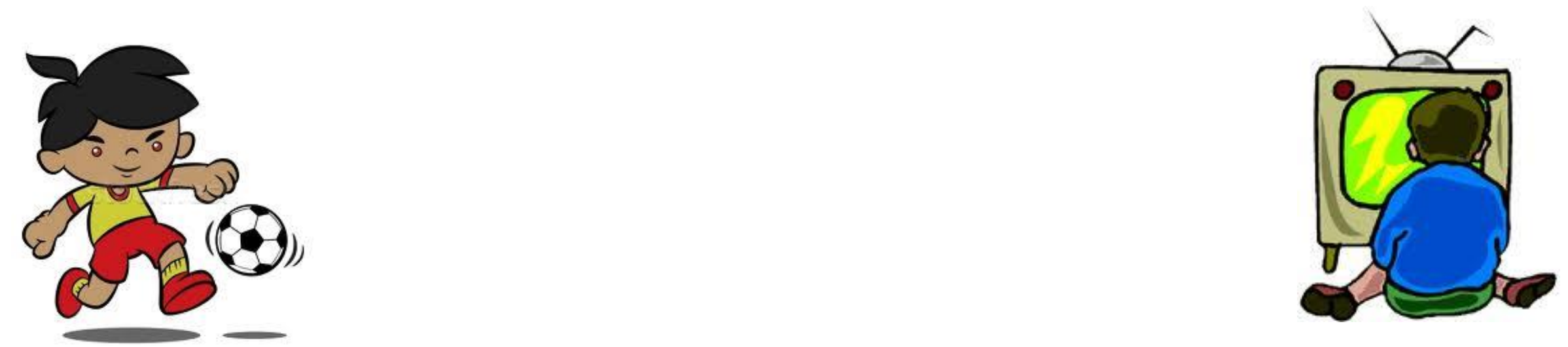


\section{Method}

- Growing Up in Ireland Study: longitudinal study to describe lives of Irish children (infant and child cohort)

- Total $\mathrm{N}=8568$ nine-year olds (49\% boys) and their parents

- Randomly selected to ensure geographical representation; $57 \%$ response

- Parental report of child PA (participation in hard exercise over a two week period-Leisure Time Exercise Questionnaire), TV time, presence of electronic equipment in the child's room, sport club membership, mobile phone ownership, activities with parents

- Social class, gender

- Objectively measured BMI 


\section{Method - Analysis}

\begin{tabular}{|l|l|}
\hline High PA & Description \\
\hline Low PA & $>9$ bouts of hard exercise over 2 weeks \\
\hline High ST & $0-8$ bouts of hard exercise over 2 weeks \\
\hline Low ST & $>3$ hours of screen time per day \\
\hline OW/Obese & $<3$ hours of screen time per day \\
\hline Normal/Underweight & $\mathrm{BMI} \geq 19.1$ boys \\
\hline
\end{tabular}

Chi squared statistics and forced entry logistic regression was used to identify factors associated with OW/obesity and with ST . Data presented as adjusted odds ratios. 


\section{Combined Categories}

To assess the combined influence of PA and ST on OW/obesity, children were categorised into one of four ST/PA groups

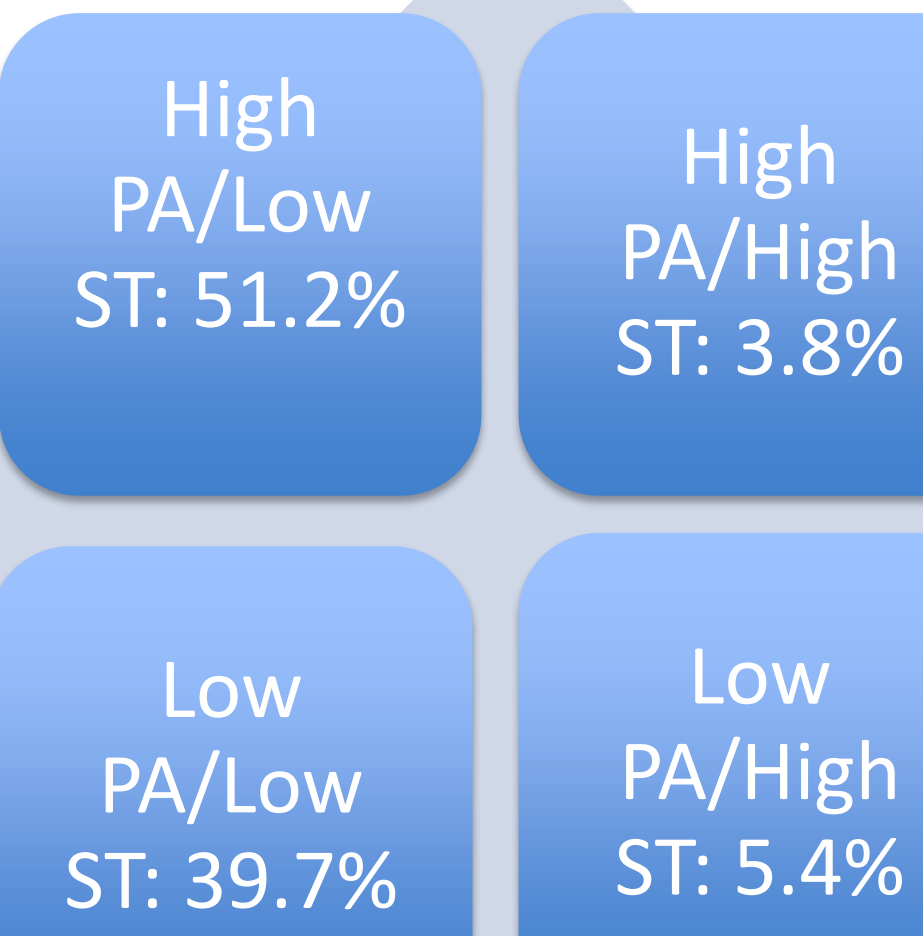

Low PA/Low ST: $39.7 \%$

Low

PA/High

ST: $5.4 \%$ 


\begin{tabular}{|c|c|c|c|c|}
\hline \multicolumn{2}{|c|}{$\begin{array}{l}\text { Predictors of } \\
\text { ow/obesity }\end{array}$} & $\begin{array}{l}\text { Overall Sample } \\
\text { Adjusted OR } \\
(95 \% \mathrm{Cl}) \\
\mathrm{n}=7035\end{array}$ & $\begin{array}{l}\text { High PA } \\
\text { Adjusted OR } \\
(95 \% \mathrm{Cl}) \\
n=3860\end{array}$ & $\begin{array}{l}\text { Low PA } \\
\text { Adjusted OR } \\
(95 \% \mathrm{Cl}) \\
\mathrm{n}=3175\end{array}$ \\
\hline Gender & $\begin{array}{l}\text { Boys } \\
\text { Girls }\end{array}$ & $\begin{array}{l}1 \\
1.41(1.25-1.60)^{\wedge}\end{array}$ & $\begin{array}{l}1 \\
1.30(1.09-1.55)^{\wedge}\end{array}$ & $\begin{array}{l}1 \\
1.51(1.27-1.80)^{\wedge}\end{array}$ \\
\hline Social Class & $\begin{array}{l}\text { SC } 1-2 \\
\text { SC } 3-4 \\
\text { SC 5-6 }\end{array}$ & $\begin{array}{l}1 \\
1.18(1.04-1.34)^{\wedge} \\
1.33(1.089-1.62)^{\wedge}\end{array}$ & $\begin{array}{l}1 \\
1.24(1.03-1.48)^{\wedge} \\
1.26(.94-1.68)\end{array}$ & $\begin{array}{l}1 \\
1.11(.93-1.33) \\
1.39(1.05-1.85)^{\wedge}\end{array}$ \\
\hline Hard Exercise & $\begin{array}{l}9+\text { times } \\
\text { None/1-2 times }\end{array}$ & $\begin{array}{l}1 \\
1.38(1.11-1.73)^{\wedge}\end{array}$ & & \\
\hline Screen Time & $\begin{array}{l}\text { None/less than } 1 \mathrm{hr} \\
1-3 \mathrm{hrs} \\
>3 \mathrm{hrs}\end{array}$ & $\begin{array}{l}1 \\
1.18(1.04-1.38)^{\wedge} \\
1.78(1.43-2.22)^{\wedge}\end{array}$ & $\begin{array}{l}1 \\
1.16(.96-1.41) \\
1.78(1.29-2.47)^{\wedge}\end{array}$ & $\begin{array}{l}1 \\
1.21(.99-1.50) \\
1.77(1.31-2.39)^{\wedge}\end{array}$ \\
\hline TV in Bedroom & $\begin{array}{l}\text { No } \\
\text { Yes }\end{array}$ & $\begin{array}{l}1 \\
1.38(1.19-1.59)^{\wedge}\end{array}$ & $\begin{array}{l}1 \\
1.32(1.07-1.61)^{\wedge}\end{array}$ & $\begin{array}{l}1 \\
1.45(1.19-1.77)^{\wedge}\end{array}$ \\
\hline Mobile Phone & $\begin{array}{l}\text { No } \\
\text { Yes }\end{array}$ & $\begin{array}{l}1 \\
1.41(1.24-1.59)^{\wedge}\end{array}$ & $\begin{array}{l}1 \\
1.56(1.31-1.86)^{\wedge}\end{array}$ & $\begin{array}{l}1 \\
1.26(1.06-1.50)^{\wedge}\end{array}$ \\
\hline $\begin{array}{l}\text { Combined } \\
\text { Categories }\end{array}$ & $\begin{array}{l}\text { Low ST/High PA } \\
\text { Low ST/Low PA } \\
\text { High ST/High PA } \\
\text { High ST/Low PA }\end{array}$ & $\begin{array}{l}1 \\
1.38(1.22-1.56)^{\wedge} \\
1.63(1.23-2.18)^{\wedge} \\
2.07(1.62-2.66)^{\wedge}\end{array}$ & & \\
\hline
\end{tabular}




\section{Results (and take home messages!)}

- RQ1 Combined influence of ST and PA on risk of OW/obesity? Compared to the reference group of low ST/high PA, children who reported low ST and low PA had a $38 \%$ increased risk ( $p<.05$ ) of OW/obesity while children who maintained high PA but high ST had a $63 \%$ increased risk ( $p<.05)$.

- Children in the high ST/low PA category were twice as likely to be $O W / o b e s e(O R=2.07)$ than the reference group.

- Reinforces findings in older children : sedentarism is a predictor of weight gain independent of PA status. This justifies efforts to reduce sedentary behaviour as part of existing public health initiatives. 
- RQ2 Is high ST a risk factor for OW/obesity even in sufficiently active children? Even in high active 9 year old children, ST a predictor of OW/obesity (>3 hours ST, OR 1.78; 13 hours, OR 1.16).

- RQ3: Correlates of high ST? Regardless of PA status, technology in bedroom and having a mobile phone associated with higher screen time. 


\section{The message for parents of 9 year olds? Learn to say 'No'!}

$31 / 8 / 11$

A NEW SURVEY of Irish parents has shown that over a third of children aged 8 now owns a mobile phone -
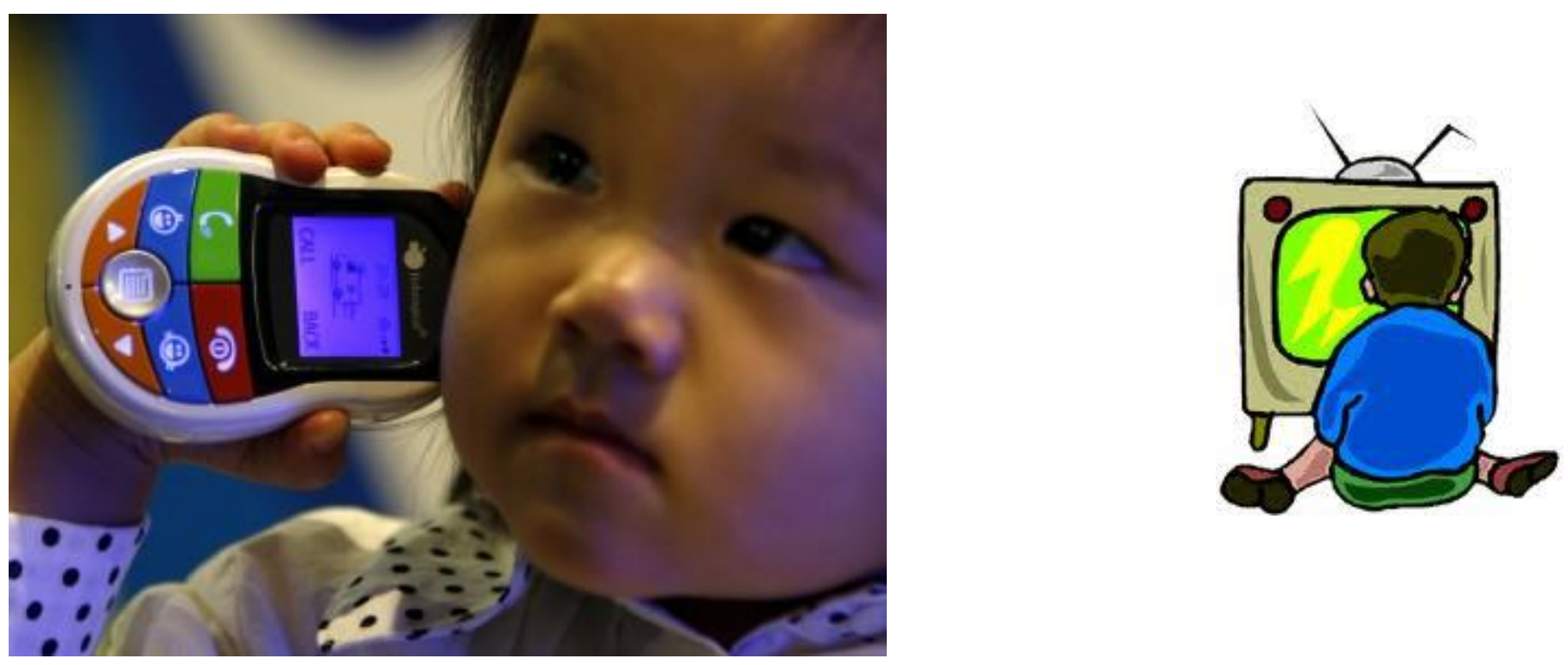